\title{
Universality of thermally assisted magnetic domain wall motion under spin torque
}

\author{
Gen Tatara* \\ PRESTO, JST, 4-1-8 Hooncho Kawaguchi, Saitama, Japan \\ and \\ Graduate School of Science, Osaka University, \\ Toyonaka, Osaka 560-0043, Japan \\ Nicolas Vernier and Jacques Ferré \\ Laboratoire de Physique des Solides, UMR CNRS 8502, \\ Bat 510, University Paris-Sud, 91405, Orsay, France
}

(Dated: June 24, 2018)

\begin{abstract}
Thermally assisted motion of magnetic domain wall under spin torque is studied theoretically. It is shown that the wall velocity $v$ depends exponentially on the spin current, $I_{\mathrm{s}}$, below the threshold value, in the same way as in a thermally activated motion driven by a force. A novel property of the spin torque driven case at low temperature is that the linear term in spin current is universal, i.e., $\ln v \sim \frac{\pi \hbar}{2 e}\left(I_{\mathrm{s}} / k_{B} T\right)$. This behavior, which is independent of pinning and material constants, could be used to confirm experimentally the spin torque as the driving mechanism.
\end{abstract}

Electric control of domain wall dynamics in nano-ferromagnets is currently under intensive study from the viewpoint of both applications and fundamental physics 1, 2, 3, 4, 5, 6, 7, 8, 9, 10, 11. In most ferromagnetic wires so far studied, domain wall motion driven by a steady current is probably related to the spin torque induced mechanism by a spin current, since domain walls are thick compared with Fermi wavelength, and thus the momentum transfer effect would be neglected [9]. Experimentally, the critical current needed to drive the wall is of most interest at present, but the wall velocity would be essential to state the involved mechanism, and also to imagine new applications. So far, the wall velocity has not been much investigated experimentally. Yamaguchi et al. performed measurements on a narrow permaloy wire [5], but the data were not precise enough to determine unanbiguously the dependence on the applied current. Clearer results were obtained recently on magnetic semiconductor wires 6, [12], where an exponential dependence of the wall velocity as function of the applied current density $(j)$ was evidenced to be $\ln v \propto j[12$. This behavior is reminiscent of a thermally assisted motion under a driving force, often found in many systems below its threshold value $13,14,15]$.

An interesting question here is whether the thermally activated motion under spin torque is indeed similar as that induced by a force. The answer is not obvious since domain wall dynamics induced by a spin torque above the threshold current has shown to behave quite differently from a force driven case 9]. In the case of a uniform magnetization resersal, Li and Zhang [16] found theoretically that spin dynamics under a spin torque are characterized by an exponential dependence of the flip rate on the spin torque, i.e., a similar behavior as in a field-driven case.

Let us consider a planar domain wall at position $X(t)$ moving in one direction in the presence of a pinning potential of harmonic type

$$
V_{\text {pin }}(X)=\frac{1}{2} M_{w} \Omega^{2} X^{2} \theta(\lambda-|X|),
$$

where $M_{w}$ is the wall mass, $\lambda$ the wall thickness, $\Omega$ the pinning frequency, and $\theta(x)$ the step function. The mass of the wall is related to the hard axis anisotropy, $K_{\perp}$, as $M_{w}=\frac{\hbar^{2} N}{K_{\perp} \lambda^{2}}\left[17\right.$ where $N \equiv 2 \lambda A / a^{3}$ is the number of spins in the wall. The coupling to the electric current is induced by the exchange interaction. For a thick wall, which is usually the case in most experiments, the domain wall is driven by a spin torque effect [9]. Introducing $\phi$ as average of the spin angle from the easy plane, the equations of motion that describes wall dynamics can be written as $[\underline{9}]$

$$
\begin{aligned}
\dot{\phi}+\alpha \frac{\dot{X}}{\lambda} & =-\frac{1}{2} \frac{\Omega^{2}}{v_{\mathrm{c}}} X \\
\dot{X}-\alpha \lambda \dot{\phi} & =v_{\mathrm{c}} \sin 2 \phi+v_{\mathrm{e}}
\end{aligned}
$$

where $v_{\mathrm{c}} \equiv K_{\perp} \lambda S /(2 \hbar), v_{\mathrm{e}} \equiv \frac{a^{3}}{2 S e} j_{\mathrm{s}}$ is the drift velocity of the spin current density $\left(j_{\mathrm{s}}\right)$, which describes the spin torque effect. $\alpha$ is the Gilbert damping parameter. Dynamics of the wall under a spin torque is calculated in terms of

${ }^{*}$ Work done at the Laboratoire de Physique des Solides, Université Paris-Sud, Orsay, France 
the dynamics of $\phi$, as pointed out in Ref. [9]. From the above set of equations we obtain a closed equation of motion for $\phi$ as

$$
\left(1+\alpha^{2}\right) M_{\phi} \ddot{\phi}=-\alpha M_{\phi} \dot{\phi}\left(\frac{\lambda \Omega^{2}}{2 v_{\mathrm{c}}}+\frac{2 v_{\mathrm{c}}}{\lambda} \cos 2 \phi\right)-\frac{N S^{2} K_{\perp}}{2}\left(\sin 2 \phi+\frac{v_{\mathrm{e}}}{v_{\mathrm{c}}}\right)
$$

where the effective mass of $\phi$ is given by [18]: $M_{\phi} \equiv N S^{2} K_{\perp} / \Omega^{2}$. We see that the potential energy for $\phi$ is given by a tilted washboard potential, $V_{\phi}=\frac{N S^{2} K_{\perp}}{2}\left(\sin ^{2} \phi+\frac{v_{\mathrm{e}}}{v_{\mathrm{c}}} \phi\right)$, and the oscillation frequency of $\phi$ around its minimum is given by $\Omega / \sqrt{1+\alpha^{2}}$ for $v_{\mathrm{e}} \simeq 0$ (see Fig. 1). We consider the case of a weak spin current (much smaller than the threshold value (i.e., $j_{\mathrm{s}} \ll j_{\mathrm{s}}^{\mathrm{cr}}, j_{\mathrm{s}}^{\mathrm{cr}} \equiv \frac{e S^{2}}{\hbar a^{3}} K_{\perp} \lambda[9]$ ), which allows to linearize the potential with respect to $j_{\mathrm{s}}$ (or $v_{\mathrm{e}}$ ). The minimum of the potential is then given by $\phi \simeq-\frac{v_{\mathrm{e}}}{2 v_{\mathrm{c}}}$, and its maxmum occurs for $\phi \simeq-\frac{\pi}{2}+\frac{v_{\mathrm{e}}}{2 v_{\mathrm{c}}}$. For $\phi$, the barrier height, $\Delta V$, thus reads

$$
\Delta V=\frac{N S^{2} K_{\perp}}{2}\left(1-\frac{\pi}{2} \frac{v_{\mathrm{e}}}{v_{\mathrm{c}}}+O\left(\frac{v_{\mathrm{e}}}{v_{\mathrm{c}}}\right)^{2}\right)
$$

Note that this barrier is not due to the pinning $(\Omega)$, but is related to the anisotropy energy. This is an important property of spin torque induced wall motion. Here we neglect the damping term in Eq. (3), considering a fast activation process. This is true if the activation process occurs in a time shorter than $\tau=\frac{2 v_{\mathrm{c}}}{\lambda \Omega^{2} \alpha}$ (for more details, see below). The transition rate from $\phi=-\frac{v_{\mathrm{e}}}{2 v_{\mathrm{c}}}$ to the next minimum $\phi \sim-\pi+\frac{v_{\mathrm{e}}}{2 v_{\mathrm{c}}}$, induced by thermal activation is thus given by

$$
\Gamma_{L}=\Omega e^{-\Delta V /\left(k_{B} T\right)}=\Omega e^{-N S^{2} K_{\perp} /\left(2 k_{B} T\right)} e^{\frac{\pi}{2} \hbar I_{\mathrm{s}} /\left(e k_{B} T\right)}
$$

where $I_{\mathrm{s}} \equiv A j_{\mathrm{s}}$ is the spin current. The hopping probability in the other direction (i.e., to the minimum at $\left.\phi \sim \pi+\frac{v_{\mathrm{e}}}{2 v_{\mathrm{c}}}\right)$ is given by

$$
\Gamma_{R}=\Omega e^{-N S^{2} K_{\perp} /\left(2 k_{B} T\right)} e^{-\frac{\pi}{2} \hbar I_{\mathrm{s}} /\left(e k_{B} T\right)}
$$

and thus, the average drift velocity of $\phi$ is given by the difference, $\Gamma \equiv\left(\Gamma_{L}-\Gamma_{R}\right)$, as $\langle\dot{\phi}\rangle \simeq-\pi \Gamma$. This last velocity is proportional to the domain wall velocity [9] (except in the extremely strong pinning case, where $V_{0}>K_{\perp} / \alpha$, if $V_{0}$ stands for the depth of the pinning potential). By use of $\dot{\phi}=-\alpha \frac{\dot{X}}{\lambda}$, after depinning (eq. (2) with $\Omega=0$ ), the wall velocity is given by

$$
v \equiv\langle\dot{X}\rangle \simeq \frac{\pi \lambda}{\alpha} \Gamma=\frac{2 \pi \Omega \lambda}{\alpha} e^{-N S^{2} K_{\perp} /\left(2 k_{B} T\right)} \sinh \frac{\pi}{2} \frac{\hbar I_{\mathrm{s}}}{e k_{B} T}
$$

It is notable that Logarithm of the velocity shows an universal dependence on $I_{\mathrm{s}}$ (i.e., it does not involve material parameters)

$$
\ln v \simeq \ln \sinh \frac{\pi}{2} \frac{\hbar I_{\mathrm{s}}}{e k_{B} T}+C(T)
$$

where $C(T)=-\frac{N S^{2} K_{\perp}}{2 k_{B} T}+\ln \frac{2 \pi \Omega \lambda}{\alpha}$ is independent of $I_{\mathrm{s}}$. At low enough temperature, $\frac{\hbar I_{\mathrm{s}}}{e k_{B} T} \gg 1$, a typical exponential dependence on spin torque is evidenced

$$
v=e^{C(T) / 2} e^{\frac{\pi}{2} \frac{\hbar I_{\mathrm{s}}}{e k_{B} T}}
$$

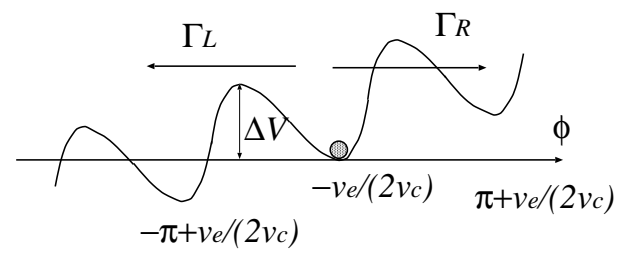

FIG. 1: Potential for $\phi$ under spin current. 
which is similar to the thermally activated wall motion under a small force. The dependence of $\ln v$ on $I_{\mathrm{S}}$ then writes

$$
\ln v \simeq \frac{\pi}{2} \frac{\hbar I_{\mathrm{s}}}{e k_{B} T}+C^{\prime}(T)
$$

with a coefficient $\frac{\pi \hbar}{2 e}$ independent on $\Omega, K_{\perp}$, nor on $\lambda\left(C^{\prime}(T) \equiv C(T)-\ln 2\right)$. This universal behavior in the thermally activated regime would be used to identify the driving mechanism (i.e., spin torque or momentum transfer).

In deriving eq. (5), we neglected the dissipation, that is justified if the disspation time scale, $\tau=\frac{2 v_{c}}{\lambda \Omega^{2} \alpha}$ is longer than the activation time, $\Gamma^{-1}=\frac{\pi \lambda}{\alpha v}$. Thus, the above statement only applies if the domain wall velocity satisfies

$$
v \gtrsim \frac{\pi}{2} \frac{(\lambda \Omega)^{2}}{v_{\mathrm{c}}}
$$

This condition is easily satisfied. For instance, for $\Omega \sim 10 \mathrm{MHz}[\underline{8}]$ and $\lambda=100 \mathrm{~nm}, \lambda \Omega=1[\mathrm{~m} / \mathrm{s}]$, while $v_{\mathrm{c}} \sim 600[\mathrm{~m} / \mathrm{s}]$ if $K_{\perp}=0.1 \mathrm{~K}$ per site, that gives $\frac{\pi}{2} \frac{(\lambda \Omega)^{2}}{v_{\mathrm{c}}} \sim 3 \times 10^{-3}[\mathrm{~m} / \mathrm{s}]$, while experiments were performed for $v \gtrsim 1[\mathrm{~m} / \mathrm{s}][\underline{5},[\underline{6}]$.

The linearization with respect to $j_{\mathrm{s}}$ in eq. (4) requires $j_{\mathrm{s}} \ll j_{\mathrm{s}}^{\mathrm{cr}}$, and this condition and eq. (11) detemine the window where the universal behavior is satisfied. This window might be wide as indicated by recent experimental observation of the exponential dependence of $v$ on $I_{\mathrm{s}}$ in GaMnAs [12] for a current density larger than $10^{9}\left[\mathrm{~A} / \mathrm{m}^{2}\right]$ at tempratures in the $86-94 \mathrm{~K}$ range.

In conclusion, we have found an universal behavior for the wall velocity in the thermally activated regime below the threshold spin current. The analysis is based on the standard theory of thermal activation, which assumes small oscillations in potential wells. The pinning plays only an essential role in introducing a finite attempt frequency, $\Omega$.

Acknowledgements:

The authors are grateful to M. Yamanouchi, D. Chiba, F. Matsukura, H. Ohno, R. Stamps, H. Kohno and J. Shibata for valuable discussion. GT thanks Monka-shou and The Mitsubishi Foundation for financial support, and the CNRS for granting his stay in France as an invited associated researcher.

[1] J. Grollier, D. Lacour, V. Cros, A. Hamzic, A. Vaurés, A. Fert, D. Adam and G. Faini, J. Appl. Phys. 92, 4825 (2002); Appl. Phys. Lett. 83, 509 (2003).

[2] M. Tsoi, R. E. Fontana, and S. S. P. Parkin Appl. Phys. Lett. 83, 2617 (2003).

[3] M. Kläui, C. A. F. Vaz, J. A. C. Bland, W. Wernsdorfer, G. Faini, E. Cambril and L. J. Heyderman, Appl. Phys. Lett. 83, 105 (2003).

[4] N. Vernier, D. A. Allwood, D. Atkinson, M. D. Cooke and R. P. Cowburn, Europhys. Lett. 65, 526 (2004).

[5] A. Yamaguchi, T. Ono, S. Nasu, K. Miyake, K. Mibu and T. Shinjo, Phys. Rev. Lett. 92077205 (2004).

[6] M. Yamanouchi, D. Chiba, F. Matsukura, and H. Ohno, Nature, 428, 539 (2004).

[7] C. K. Lim, T. Devolder, C. Chappert, J. Grollier, V. Cros, A. Vaurś, A. Fert, and G. Faini, Appl. Phys. Lett. 84, 2820 (2004).

[8] E. Saitoh, H. Miyajima, T. Yamaoka and G. Tatara, preprint.

[9] G. Tatara and H. Kohno, Phys. Rev. Lett. 92 086601(2004).

[10] A. Thiaville, Y. Nakatani, J. Miltat, and N. Vernier, J. Appl. Phys. 95, 7049 (2004).

[11] Z. Li and S. Zhang, Phys. Rev. Lett. 92, 207203 (2004); S. Zhang and Z. Li, Phys. Rev. Lett. 93, 127204 (2004).

[12] M. Yamanouchi, D. Chiba, F. Matsukura and H. Ohno, private communication.

[13] P. Bruno, G. Bayreuther, P. Beauvillain, C. Chappert, G. Lugert, D. Renard, J. P. Renard and J. Seiden, J. Appl. Phys. 68, 5759 (1990).

[14] J. Ferré, in Spin Dynamics in Confined Magnetic Structures I, Ed. by B. Hillebrands, Springer, Topics in Appl. Phys. 83, 127 (2002).

[15] M. Muller, D. A. Gorokhov and G. Blatter, Phys. Rev. B63, 184305 (2001).

[16] Z. Li and S. Zhang Phys. Rev. B69, 134416 (2004).

[17] S. Chikazumi, Physics of Ferromagnetism (Oxford university press, 1997).

[18] S. Takagi and G. Tatara, Phys. Rev. B54, 9920 (1996). 